\title{
C9orf72 deficiency promotes motor deficits of a C9ALS/FTD mouse model in a dose- dependent manner
}

\author{
Qiang Shao ${ }^{\dagger}$, Chen Liang ${ }^{\dagger}$, Qing Chang, Wei Zhang, Mei Yang and Jian-Fu Chen ${ }^{*}$ (1)
}

Keywords: C9orf72, Motor deficits, C9ALS/FTD, Mice

G4C2 hexanucleotide repeat expansions in the first intron of C9ORF72 are the most common cause of familial amyotrophic lateral sclerosis (ALS) and frontotemporal dementia (FTD) (collectively, C9ALS/FTD) $[4,6,11,14]$. Haploinsufficiency (loss-of-function) of C9ORF72 protein is a key proposed disease mechanism which may act in parallel with gain-of-function mechanisms, including toxic RNAs from repeat transcription and dipeptide repeat proteins (DPRs) from repeat-associated non-AUG (RAN) translation [5, 9, 17]. However, the effect of C9orf72 deficiency in the background of gain-of-function has not been examined in vivo. Neither heterozygous nor homozygous knockout (KO) of C9orf72 in neurons leads to motor deficits in mice [8]. Recently, gain-of-function mouse models were generated using a C9ORF72 bacterial artificial chromosome (BAC) from C9ALS/FTD patient DNA under the control of the endogenous regulatory elements. Interestingly, three out of four of these $C 9-B A C$ transgenic mice did not develop motor behavior deficits, even at advanced ages [7, 12, 13]. Since these C9-BAC mouse models contain elevated C9orf72 proteins from the endogenous mouse gene, we hypothesized that $\mathrm{C} 9$ orf72 provides neuroprotective effects against motor deficits in $C 9-B A C$ mice.

To test this hypothesis and investigate the in vivo significance of C9orf72 haploinsufficiency, we crossed $C 90 \mathrm{rf} 72^{+-}$mice with $C 9-B A C$ mice and examined the consequences of $\mathrm{C} 9$ orf72 protein dose reduction (loss-of-function) in the background of C9-BAC (gain-of-function). We found that C9orf72 loss and haploinsufficiency exacerbate motor behavior deficits in

\footnotetext{
* Correspondence: jianfu@usc.edu

${ }^{\dagger}$ Qiang Shao and Chen Liang contributed equally to this work.

Center for Craniofacial Molecular Biology, University of Southern California (USC), Los Angeles, CA 90033, USA
}

a dose-dependent manner, and this occurs early in the course of pathogenesis (4 months of age). Among the four published $C 9-B A C$ mouse models, we selected the one with motor deficits (we refer to this C9orf $72 B A C^{T g}$ + model as the C9-BAC line here) [10]. To reduce C9orf72 protein levels at different doses, we crossed $C 90 r f 72^{+/-}$and $C 9-B A C$ mice for two generations. We isolated proteins from brain tissues and confirmed the expected C9orf72 protein dose reduction (Fig. 1a, Additional file 1: Figure S1A). The unchanged protein level of Atg101, which is associated with the C9orf72/Smcr8 complex based on our previous study [16], suggests the specificity of C9orf72 reduction (Fig. 1a, Additional file 1: Figure S1A).

To study effects of C9orf 72 deficiency on the motor behaviors of $C 9-B A C$ mice, we monitored a cohort of mice [20 WT (10 females + 10 males), $18 C 9-B A C$ (11 females +7 males), 26 C9orf $72^{+-} ; C 9-B A C$ (14 females +12 males), and 19 C9orf $72^{-/} ; C 9-B A C$ (10 females +9 males)]. We excluded $C 9$ orf $72^{+/-}$and $C 9$ orf $72^{-/-}$mice for the following reasons: $C 9$ orf 72 heterozygous and homozygous $\mathrm{KO}$ mice exhibited no neurodegeneration and motor deficits based on previous studies [8]; complete deletion of C9orf72, which does not occur in C9ALS/FTD patients, led to autoimmune disorders and reduced survival in mice [1], which may complicate large-scale behavior and survival studies. We found that there were no significant differences among the four tested groups in their survival around 4 months, when behaviors were assessed. They also exhibited similar body weights, taking the sex of the mice into account (Additional file 1: Figure S1B-1C). To examine their general anxiety levels, we performed an open field test [3]. C9-BAC mice with different C9orf72 levels behaved similarly in total distance traveled, 


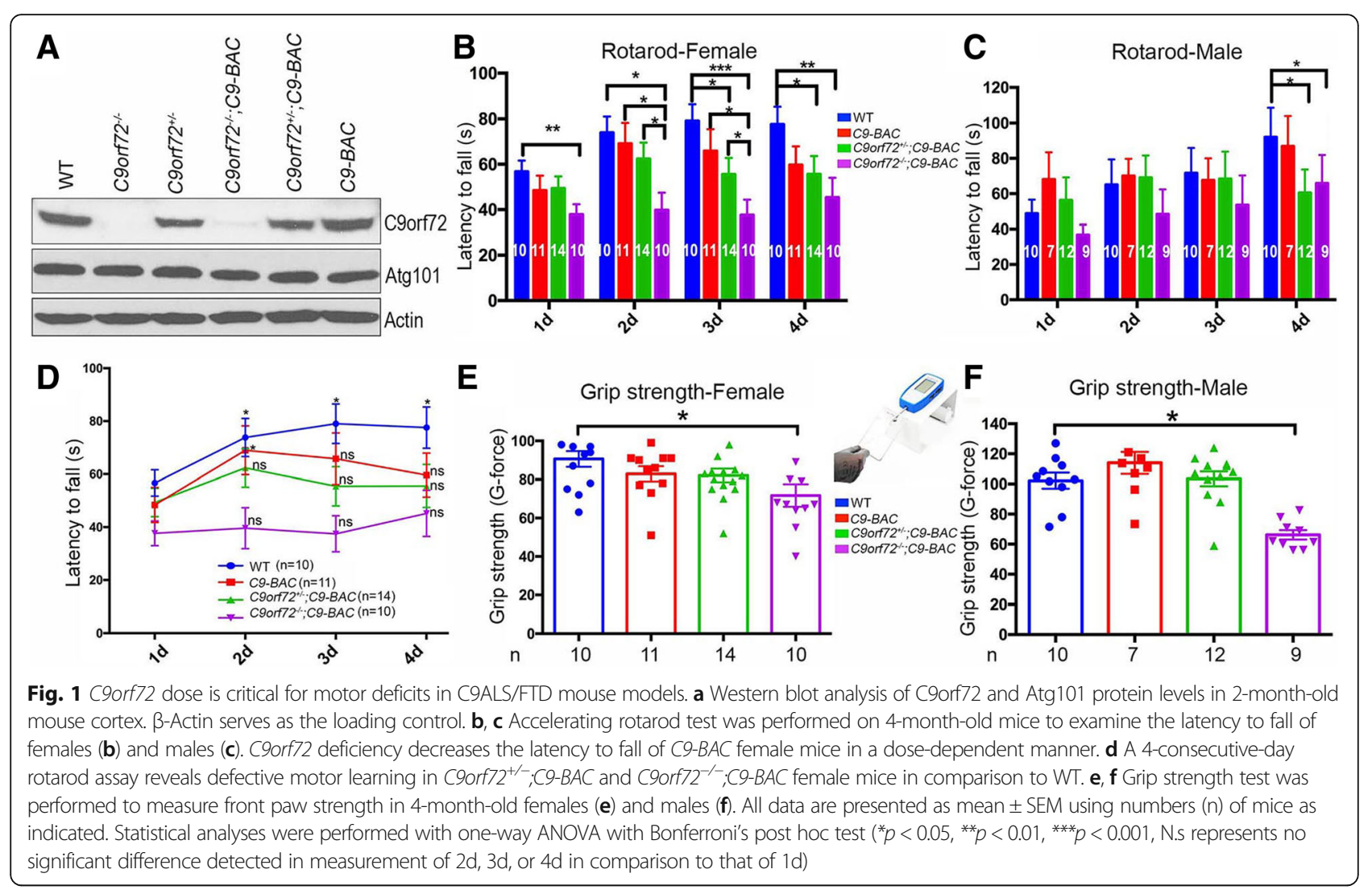

distance traveled in the center, and time spent in the center (Additional file 1: Figure S1E-1G).

We next examined their motor coordination and balance using an accelerating (4-40 rpm in $5 \mathrm{~min}$ ) rotarod test. Mice were given five trials per day, with an inter-trial interval of $20 \mathrm{~min}$, for 4 consecutive days. A C9orf72 dose-dependent decrease in latency to fall was detected in C9-BAC female mice (Fig. 1b), and in $C 9-B A C$ male mice on day 4 of the rotarod assay (Fig. 1c). These results suggest that motor coordination is sensitive to C9orf72 protein levels in C9-BAC mice. We further analyzed motor learning in female mice. WT mice exhibited an increase in latency to fall over the course of 4 consecutive days, indicating active motor learning (Fig. 1d). Latency to fall of C9-BAC mice was increased on day 2 but dropped on days 3 and 4 (Fig. 1d). Importantly, there was no increase in latency to fall from day 1 to day 4 in $C 9$ orf $72^{+/-} ; C 9-B A C$ and $C 9$ orf $72^{-/-} ; C 9-B A C$ animals (Fig. 1d). These results suggest that $C 9$ orf 72 deficiency impaired motor coordination and motor learning of C9-BAC mice in a dose-dependent manner.

To examine motor strength, we measured forearm grip strength and found that it was significantly reduced in both male and female $C 9$ orf $72^{-/-} ; C 9 B A C$ animals compared to other genotypes (Fig. 1e, f). Lastly, we measured the maximal speed at which each animal fell from the rotarod device. Results showed that
C9orf72 deficiency, in a dose-dependent manner, decreased the maximum speed at which $C 9-B A C$ mice fell (Additional file 1: Figure S1H, S1I), which is consistent with the data on their latency to fall.

The rotarod assay revealed more evident motor impairment in female mice than in male mice. This could be due to toxic gain-of-function since $C 9-B A C$ female mice exhibited earlier and more pronounced abnormalities than male mice [10]. It will be important to examine using similar cohorts of mice whether motor neurons (MNs) degenerate or reduce in number in a C9orf72 dosedependent manner and whether these deficits correlate with the observed motor behavior deficits. Future studies should also investigate whether C9orf72 exhibits dose-dependent effects in the three other C9-BAC mouse models $[7,12,13]$. It will be informative to examine the effects of C9orf72 deficiency in the background of adeno-associated virus (AAV)-mediated G4C2 repeat expression [2]. Our study indicates that C9orf72 haploinsufficiency contributes to disease onset in a mouse model by exacerbating the pathogenic effects of RNA/DPR-mediated neurotoxicity. Together with a recent report on patient iPSC-derived MNs [15], this study suggests indeed that we should focus more on the combination of lossand toxic gain-of-function. Together, for the first time, our mouse genetic studies showed that C9orf 72 loss or haploinsufficiency in a gain-of-function mouse model of 
C9ALS/FTD exacerbate motor behavior deficits in a dose-dependent manner, demonstrating the importance of C9orf72 haploinsufficiency in vivo.

\section{Additional file}

Additional file 1: Figure S1. Characterization of C9-BAC mice with C9orf72 dose reduction. (A) Quantification of C9orf72/Atg101 protein levels. Data are presented as mean \pm SEM from three independent experiments. ( $B_{\text {, }}$ C) Body weight of female (B) and male (C) mice at 4 months of age. (D-G) Open field test was performed on 4-month-old mice to examine the total distance traveled $(E)$, distance traveled in the center $(F)$, and percentage of time spent in the center $(\mathrm{G}) .(\mathrm{H}, \mathrm{l})$ Quantification of the maximal $\mathrm{g}$ force from five trials of rotarod assay. C9orf72 deficiency decreases the maximal $g$ force of C9-BAC female mice in a dose-dependent manner. All data are presented as mean \pm SEM using numbers $(n)$ of mice as indicated. Statistical analyses were performed with one-way ANOVA with Bonferroni's post hoc test $\left({ }^{*} p<0.05,{ }^{* *} p<0.01,{ }^{* * *} p<0.001\right.$, n.s represents no significant difference detected). (PDF $2855 \mathrm{~kb}$ )

\section{Acknowledgments}

We thank Chen laboratory colleagues for stimulating discussions. We are grateful for Bridget Samuels's critical reading of the manuscript. Chen laboratory is supported by funds from the Associate Dean of Research Fund from the Center for Craniofacial Molecular Biology, Herman Ostrow School of Dentistry at the University of Southern California, and grants R01NS097231 (J.C.) and R01NS096176 (J.C.) from the National Institute of Health.

\section{Authors' contributions}

QS, QC, CL performed all behavior studies and statitical analyses. MY and WZ helped with the manuscript writing. J-FC designed and interpreted the experiments and wrote the manuscript. All authors read and approved the final manuscript.

\section{Competing interests}

The authors declare that they have no competing interests.

\section{Publisher's Note}

Springer Nature remains neutral with regard to jurisdictional claims in published maps and institutional affiliations.

Received: 4 February 2019 Accepted: 23 February 2019

Published online: 04 March 2019

References

1. Burberry A, Suzuki N, Wang J-Y, Moccia R, Mordes DA, Stewart MH et al (2016) Loss-of-function mutations in the C9ORF72 mouse ortholog cause fatal autoimmune disease. Sci Transl Med 8:347ra93-347ra93

2. Chew J, Gendron TF, Prudencio M, Sasaguri H, Zhang Y-J, CastanedesCasey $\mathrm{M}$ et al (2015) Neurodegeneration. C9ORF72 repeat expansions in mice cause TDP-43 pathology, neuronal loss, and behavioral deficits. Science. 348:1151-1154

3. Crawley JN (1999) Behavioral phenotyping of transgenic and knockout mice: experimental design and evaluation of general health, sensory functions, motor abilities, and specific behavioral tests. Brain Res 835:18-26

4. DeJesus-Hernandez M, Mackenzie IR, Boeve BF, Boxer AL, Baker M, Rutherford NJ et al (2011) Expanded GGGGCC hexanucleotide repeat in noncoding region of C9ORF72 causes chromosome 9p-linked FTD and ALS. Neuron. 72:245-256

5. Gao F-B, Almeida S, Lopez-Gonzalez R (2017) Dysregulated molecular pathways in amyotrophic lateral sclerosis-frontotemporal dementia spectrum disorder. EMBO J EMBO Press 36:2931-2950

6. Gijselinck I, Van Langenhove T, van der Zee J, Sleegers K, Philtjens S, Kleinberger $\mathrm{G}$ et al (2012) A C9orf72 promoter repeat expansion in a Flanders-Belgian cohort with disorders of the frontotemporal lobar degeneration-amyotrophic lateral sclerosis spectrum: a gene identification study. Lancet Neurol 11:54-65
7. Jiang J, Zhu Q, Gendron TF, Saberi S, McAlonis-Downes M, Seelman A et al (2016) Gain of toxicity from ALS/FTD-linked repeat expansions in C9ORF72 is alleviated by antisense oligonucleotides targeting GGGGCC-containing RNAs. Neuron. 90:535-550

8. Koppers M, Blokhuis AM, Westeneng H-J, Terpstra ML, Zundel CAC, Vieira de Sá R et al (2015) C9orf72 ablation in mice does not cause motor neuron degeneration or motor deficits. Ann Neurol 78:426-438

9. Ling S-C, Polymenidou M, Cleveland DW (2013) Converging mechanisms in ALS and FTD: disrupted RNA and protein homeostasis. Neuron. 79:416-438

10. Liu Y, Pattamatta A, Zu T, Reid T, Bardhi O, Borchelt DR et al (2016) C9orf72 BAC mouse model with motor deficits and neurodegenerative features of ALS/FTD. Neuron. 90:521-534

11. Majounie E, Renton AE, Mok K, Dopper EGP, Waite A, Rollinson S et al (2012) Frequency of the C9orf72 hexanucleotide repeat expansion in patients with amyotrophic lateral sclerosis and frontotemporal dementia: a cross-sectional study. Lancet Neurol 11:323-330

12. O'Rourke JG, Bogdanik L, Muhammad AKMG, Gendron TF, Kim KJ, Austin A et al (2015) C9orf72 BAC transgenic mice display typical pathologic features of ALS/FTD. Neuron. 88:892-901

13. Peters OM, Cabrera GT, Tran H, Gendron TF, McKeon JE, Metterville J et al (2015) Human C9ORF72 Hexanucleotide expansion reproduces RNA foci and dipeptide repeat proteins but not neurodegeneration in BAC transgenic mice. Neuron. 88:902-909

14. Renton AE, Majounie E, Waite A, Simón-Sánchez J, Rollinson S, Gibbs JR et al (2011) A hexanucleotide repeat expansion in C9ORF72 is the cause of chromosome 9p21-linked ALS-FTD. Neuron. 72:257-268

15. Shi Y, Lin S, Staats KA, Li Y, Chang W-H, Hung S-T et al (2018) Haploinsufficiency leads to neurodegeneration in C9ORF72 ALS/FTD human induced motor neurons. Nat Med Nature Publishing Group 24:313-325

16. Yang M, Liang C, Swaminathan K, Herrlinger S, Lai F, Shiekhattar R et al (2016) A C9ORF72/SMCR8-containing complex regulates ULK1 and plays a dual role in autophagy. Sci Adv American Association for the Advancement of Science 2:e1601167-e1601167

17. Zu T, Pattamatta A, LPW R (2018) Repeat-Associated Non-ATG Translation in Neurological Diseases. Cold Spring Harb Perspect Biol. Cold Spring Harbor Lab 10:a033019

\section{Ready to submit your research? Choose BMC and benefit from:}

- fast, convenient online submission

- thorough peer review by experienced researchers in your field

- rapid publication on acceptance

- support for research data, including large and complex data types

- gold Open Access which fosters wider collaboration and increased citations

- maximum visibility for your research: over $100 \mathrm{M}$ website views per year

At $\mathrm{BMC}$, research is always in progress.

Learn more biomedcentral.com/submission 